\title{
Comparison of phase-field models of fracture coupled with plasticity
}

Alessi R., Ambati M., Gerasimov T., Vidoli S., De Lorenzis L.

\begin{abstract}
In the last few years several authors have proposed different phasefield models aimed at describing ductile fracture phenomena. Most of these models fall within the class of variational approaches to fracture proposed by Francfort and Marigo [13]. For the case of brittle materials, the key concept due to Griffith consists in viewing crack growth as the result of a competition between bulk elastic energy and surface energy. For ductile materials, however, an additional contribution to the energy dissipation is present and is related to plastic deformations. Of crucial importance for the performance of the modeling approaches is the way the coupling is realized between plasticity and phase field evolution. Our aim is a critical revision of the main constitutive choices underlying the available models and a comparative study of the resulting predictive capabilities.
\end{abstract}

\section{Introduction}

Both theoretical and numerical investigations have proved the efficiency of gradient damage models in modeling the nucleation and evolution of cracks

\footnotetext{
Alessi Roberto

Department of Mathematics, SAPIENZA Universitá di Roma, Piazzale Aldo Moro 5, 00185 Roma, Italy

e-mail: roberto.alessi@uniroma1.it

Ambati Marreddy, Gerasimov Tymofiy and De Lorenzis Laura

Institute of Applied Mechanics, Technische Universität Braunschweig, Bienroder Weg 87, 38106 Braunschweig, Germany

e-mail: m.ambati@tu-braunschweig.de, t.gerasimov@tu-braunschweig.de and

1.delorenzis@tu-braunschweig.de

Vidoli Stefano, Department of Structural and Geotechnical Engineering, SAPIENZA

Universitá di Roma, Via Eudossiana 18, 00184 Roma, Italy

e-mail: stefano.vidoli@uniroma1.it
} 
in brittle materials. Under suitable choices of the constitutive functions, it is possible to prove that gradient damage models $\Gamma$-converge to Griffith's model of brittle fracture in the limit to zero of an internal length parameter. These models are also referred to as phase-field models as they contain one or more internal variables to describe the material degradation.

In brittle materials, since the creation of a crack surface is the main source of dissipation, a scalar field is usually sufficient to represent the material level of degradation and to account for the surface energy of Griffith's theory. Ductile fracture is, instead, associated to more complex processes: large energy absorption, due to the nucleation and coalescence of micro-voids accompanied by extensive plastic deformations precede the actual formation of cracks. Hence, measures of the current and accumulated plastic strains seem natural candidates to complement the scalar damage in the internal material description.

Several authors have recently proposed gradient damage models coupled to plastic effects. The main difficulty is the simultaneous presence and competition of two dissipative terms, due to plastic flow and to crack growth. In particular, we cite the models by [1, 2], [9], [12], [14], [17], [4, 5]. Our purpose is to review these recent contributions and to attempt a comparative study of their performance in describing the main aspects of ductile fracture. For the models under consideration, the only rigorous result of $\Gamma$-convergence obtained so far concerns the model [1, 2], see [11].

Limiting ourselves to infinitesimal deformations, we formulate a common variational setting encompassing all the considered models, in which we are able to highlight the different choices for the main constitutive functions. Then, we perform a numerical comparison of all models for a standard uniaxial tension test. The paper is organised as follows. Sec. 2 sets the variational framework. Sec. 3 overviews and comments on the specific choices made in the various models, whereas Sec. 4 reports numerical comparisons. Conclusions are drawn in Sec. 5.

\section{A general framework for phase-field models of fracture coupled with plasticity}

In this section, we present a unified variational setting which encompasses several, recently proposed phase-field fracture models coupled with plasticity. Assuming that external actions are sufficiently smooth in time and inertial effects are negligible, we consider the energetic formulation for rateindependent problems [18]. This relies on three simple energetic principles, namely an energy balance, a dissipation inequality and a stability criterion, from which all the standard governing equations can be easily obtained. In such a context, for each model under consideration, it is sufficient to define 
the basic state variables and to introduce the total internal energy density, a state function which includes both potential and dissipative contributions.

\subsection{State variables}

Let $\Omega$ be an open bounded domain in $\mathbb{R}^{N}$ representing the reference configuration of a body, with Neumann and Dirichlet boundaries $\partial \Omega_{N}$ and $\partial \Omega_{D}$, Fig. 1. The state of each point $\boldsymbol{x} \in \Omega$ is defined by the variables in Tab. 1 .

\begin{tabular}{lll}
\hline state variables & type \\
\hline $\boldsymbol{u}$ & displacement & observable \\
$\boldsymbol{\varepsilon}$ & total strain & \\
\hline $\boldsymbol{p}$ & plastic strain (trace free, $\operatorname{tr} \boldsymbol{p}=0)$ & \\
$\alpha$ & scalar hardening variable $($ accumulated & internal \\
$\quad$ plastic strain, irreversible) & \\
$\nabla \alpha$ & gradient of hardening variable & \\
\hline$d$ & scalar damage $($ irreversible $)$ & internal \\
$\nabla d$ & gradient of damage & \\
\hline
\end{tabular}

Table 1: State variables.

Only the displacement field $\boldsymbol{u}(\boldsymbol{x}, t)$, the plastic strain field $\boldsymbol{p}(\boldsymbol{x}, t)$ and the damage field $d(\boldsymbol{x}, t)$ are independent. The infinitesimal strain is the symmetric gradient of the displacement field, and the hardening variable, identified in this context with the accumulated plastic strain, is given by

$$
\alpha(\boldsymbol{x}, t):=k_{N} \int_{0}^{t}\|\dot{\boldsymbol{p}}\| \mathrm{d} \tau, \quad k_{N}= \begin{cases}1, & \text { if } N=1 \\ \frac{N-1}{N}, & \text { otherwise }\end{cases}
$$

The scalar damage field is bounded, since we assume $d \in[0,1] ; d=0$ means a sound material and $d=1$ a fully damaged material. This field must satisfy the following irreversibility condition to prevent healing effects, as first introduced in [7] and [16]

$$
\dot{d} \geq 0, \quad \forall t \text { and } \forall \boldsymbol{x} .
$$

In the following subsections, we briefly introduce the total energy densities of gradient damage models, traditionally used to describe brittle or quasi-brittle fracture phenomena, and of gradient plasticity models, traditionally used to describe plastic hinges or shear bands. Their combination is discussed in Sec. 2.4, whereas Sec. 2.5 summarises the governing equations and evolution laws. 


\subsection{Gradient damage models}

The total internal energy density is the sum of two terms

Fig. 1 Schematic representation of the problem.

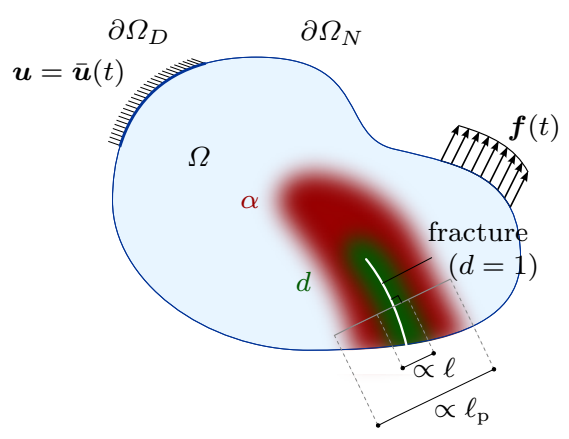

$$
W_{\mathrm{D}}(\varepsilon, d, \nabla d):=\underbrace{\mathrm{g}(d) \psi_{\mathrm{e}}(\varepsilon)}_{\begin{array}{c}
\text { free en. } \\
\text { (elastic pot.) }
\end{array}}+\underbrace{\Delta_{\mathrm{f}}(d, \nabla d)}_{\text {damage diss. }}
$$

the first representing the free energy energy density and the second the local dissipated damage work. Here, $\psi_{\mathrm{e}}(\varepsilon)$ is the elastic energy density of a sound material. The scalar degradation function $\mathrm{g}(d)$ models the deterioration due to the microcracks nucleation and growth. This function is assumed to satisfy the following conditions

$$
\mathrm{g}(1)=1, \quad \mathrm{~g}(0)=0, \quad \mathrm{~g}^{\prime}(d) \leq 0
$$

In almost all the considered models, this function is assumed in the form

$$
\mathrm{g}(d):=(1-d)^{2}
$$

The damage dissipation term accounts for the energy lost during the cracking process. A widely adopted expression ${ }^{1}$ is

1 In the gradient damage context, a different expression is often considered instead of (7), namely

$$
\Delta_{\mathrm{d}}(d, \nabla d):=\mathrm{w}(d)+\frac{1}{2} \ell_{\mathrm{d}}^{2} \mathrm{w}_{1}|\nabla d|^{2}
$$

The constitutive functions and constants are linked by the following relations

$$
\ell_{\mathrm{d}}=\sqrt{2} \ell, \quad \mathrm{w}(1)=: \mathrm{w}_{1}=G_{\mathrm{c}} /\left(\ell c_{\omega}\right), \quad \mathrm{w}(d) / \mathrm{w}_{1}=\omega(d)
$$




$$
\Delta_{\mathrm{f}}(d, \nabla d):=\frac{G_{\mathrm{c}}}{c_{\omega}}\left(\frac{\omega(d)}{\ell}+\ell|\nabla d|^{2}\right)
$$

where the constant $G_{\mathrm{c}}$ represents the fracture toughness of the material, that is the energy dissipated to create a crack of unitary area. As follows, the two most commonly used models will be considered and termed AT- 1 and AT-2 (AT refers to the Ambrosio-Tortorelli functional [6]):

$$
\omega(d)=\left\{\begin{array}{ll}
d, & \text { AT- } 1 \\
d^{2}, & \text { AT-2 }
\end{array} \quad \text { and } \quad c_{\omega}:=4 \int_{0}^{1} \sqrt{\omega(\beta)} \mathrm{d} \beta= \begin{cases}8 / 3, & \text { AT }-1 \\
2, & \text { AT-2 }\end{cases}\right.
$$

The main difference between these two models is that AT-1, due to the linear term, owns an elastic stage before the onset of fracture, while with AT-2 damage starts to evolve as soon as the material is loaded.

Phase-field models characterized by total energies in the form (2) have been rigorously proved to $\Gamma$-converge to Griffith's brittle fracture model. As the internal length $\ell$ tends to zero, the phase-field variable, which can be mechanically interpreted as a damage variable [15], localizes towards the fracture path and the global minima of the phase-field energy functional tend towards those of the energy functional of Griffith's brittle fracture [10].

\subsection{Gradient plasticity models}

The total internal energy density is taken as

$$
W_{\mathrm{P}}(\boldsymbol{\varepsilon}, \boldsymbol{p}, \alpha, \nabla \alpha):=\underbrace{\overbrace{\psi_{\mathrm{e}}(\boldsymbol{\varepsilon}-\boldsymbol{p})}^{\text {elastic pot. }}+\overbrace{\psi_{\mathrm{p}}(\alpha, \nabla \alpha)}^{\text {plastic hard. }}}_{\text {free en. }}+\underbrace{\Delta_{\mathrm{p}}(\alpha)}_{\text {plastic diss. }},
$$

Here we consider, for sake of brevity, only the Hencky-Mises plastic model. The first term represents the elastic energy of an undamaged material and depends on the elastic strain $\boldsymbol{\varepsilon}-\boldsymbol{p}$. The second free energy term represents a non-local isotropic hardening contribution and reads

$$
\psi_{\mathrm{p}}(\alpha, \nabla \alpha):=\frac{1}{2} H \alpha^{2}+\mathrm{f}(\alpha)+\frac{1}{2} \ell_{\mathrm{p}}^{2}|\nabla \alpha|^{2},
$$

the first two terms being, respectively, the linear and non-linear hardening contributions while the last term is a gradient plasticity energy source. $H$ is the hardening modulus while $\ell_{\mathrm{p}}$ is an internal material length governing the plastic localisation band width. The last term in (9) represents the plastic dissipated work and is given by 


$$
\Delta_{\mathrm{p}}(\alpha):=\sigma_{\mathrm{p}} \alpha,
$$

with $\sigma_{\mathrm{p}}$ as the plastic yield stress.

\subsection{Gradient damage models coupled with plasticity}

This section presents a gradient damage model coupled with plasticity as a rational merging of the two basic models presented in the previous subsections. Starting from total energy densities (2) and (9), the following coupled total internal energy density is postulated

$$
\begin{aligned}
& W_{\mathrm{PD}}(\boldsymbol{\varepsilon}, \boldsymbol{p}, d, \nabla d, \alpha, \nabla \alpha):=\underbrace{\psi(\varepsilon, \boldsymbol{p}, d, \alpha, \nabla \alpha)}_{\text {free energy }}+\underbrace{\Delta(d, \nabla d, \alpha)}_{\text {dissipated work }}= \\
& =\underbrace{\mathrm{g}(d) \psi_{\mathrm{e}}(\varepsilon-\boldsymbol{p})}_{\mathbf{E}}+\underbrace{\mathrm{h}(d) \psi_{\mathrm{p}}(\alpha, \nabla \alpha)}_{\mathbf{H}}+\underbrace{\Delta_{\mathrm{f}}(d, \nabla d)}_{\mathrm{F}}+\underbrace{\mathrm{p}(d) \Delta_{\mathrm{p}}(\alpha)}_{\mathrm{P}} .
\end{aligned}
$$

The first term (E) represents the elastic potential and its expression is obtained by combining the elastic potential of (2) and (9) with the degradation function $\mathrm{g}(d)$ satisfying (3). The second term $(\mathbf{H})$ represents the isotropic plastic hardening contribution supposed to be affected by a damage degradation function $\mathrm{h}(d)$ satisfying the conditions in (3), with $\psi_{\mathrm{p}}(\cdot)$ defined in (10). The third term $(\mathbf{F})$ is the fracture energy defined in (7), not directly affected by plasticity. Finally, $(\mathbf{P})$ is a damage-plasticity coupled dissipation term which contains not only the entire source of plastic dissipation but also a contribution to the damage dissipation, see also [3]. Its expression is obtained by penalizing the plastic dissipation (11) by another damage-dependent degradation function $\mathrm{p}(d)$ satisfying again (3). The stress is defined as

$$
\boldsymbol{\sigma}:=\partial_{\boldsymbol{\varepsilon}} \psi=\mathrm{g}(d)(\boldsymbol{\varepsilon}-\boldsymbol{p})
$$

The assumption (12) for the total energy encompasses all the models under consideration as special cases.

\subsection{Governing equations and evolution laws}

According to the energetic formulation, the evolution of a rate-independent system is simply governed by three energetic principles: an energy balance, a 
dissipation inequality and a stability criterion. Moreover, some explicit irreversibility conditions may be prescribed, such as here (ir). For the purposes of the present work and thanks to the regularity of the energetic functionals, it is sufficient to consider the first-order energy balance condition and the first-order directional stability condition.

Then, the evolution problem consists in finding a process $(\boldsymbol{u}, \boldsymbol{p}, \alpha, d)_{t}$, satisfying at each instant $t$ the boundary conditions, the energy balance, dissipation inequality and the first-order stability. For a detailed description of these equations see e.g. [3]. For the present model derived governing equations are summarised in Tab. 2. 


\begin{tabular}{|c|c|c|}
\hline \multicolumn{3}{|c|}{ equilibrium } \\
\hline $\begin{array}{l}\text { equilibrium } \\
\text { equations }\end{array}$ & $\operatorname{div} \boldsymbol{\sigma}+\boldsymbol{b}=\mathbf{0}, \quad \forall \boldsymbol{x} \in \Omega$ & $(14)$ \\
\hline $\begin{array}{l}\text { boundary } \\
\text { conditions }\end{array}$ & $\begin{cases}\boldsymbol{\sigma} \boldsymbol{n}=\boldsymbol{f}, & \forall \boldsymbol{x} \in \partial \Omega_{N} \\
\boldsymbol{\sigma} \boldsymbol{n}=\boldsymbol{f}_{r}, & \forall \boldsymbol{x} \in \partial \Omega_{D}\end{cases}$ & $(15)$ \\
\hline \multicolumn{3}{|c|}{ plasticity conditions } \\
\hline KKT system & $\left\{\begin{array}{l}f_{\mathrm{p}}(\boldsymbol{\sigma}, \alpha, d) \leq 0 \\
\dot{\alpha} \geq 0 \\
f_{\mathrm{p}}(\boldsymbol{\sigma}, \alpha, d) \dot{\alpha}=0\end{array} \quad, \quad \forall \boldsymbol{x} \in \Omega\right.$ & $(16)$ \\
\hline flow rule & $\begin{array}{l}\boldsymbol{p}=\dot{\alpha} \boldsymbol{n}_{\boldsymbol{\sigma}^{\prime}}, \quad \forall \boldsymbol{x} \in \Omega \\
\left(\boldsymbol{n}_{\boldsymbol{\sigma}^{\prime}}=\text { deviatoric stress direction }\right)\end{array}$ & (17) \\
\hline yield surface & $f_{\mathrm{p}}(\boldsymbol{\sigma}, \alpha, d):=$ & $(18)$ \\
\hline & $|\boldsymbol{\sigma}|-\mathrm{h}(d)\left(H \alpha+\mathrm{f}^{\prime}(\alpha)-\ell_{\mathrm{p}}^{2} \triangle \alpha\right)-\mathrm{p}(d) \sigma_{\mathrm{p}}$ & \\
\hline $\begin{array}{l}\text { boundary } \\
\text { condition }\end{array}$ & $\nabla \alpha \cdot \boldsymbol{n}=0, \quad \forall \boldsymbol{x} \in \partial \Omega$ & (19) \\
\hline \multicolumn{3}{|c|}{ damage conditions } \\
\hline KKT system & $\left\{\begin{array}{l}f_{\mathrm{d}}(\varepsilon, \boldsymbol{p}, \alpha, d) \leq 0 \\
\dot{d} \geq 0 \\
f_{\mathrm{d}}(\varepsilon, \boldsymbol{p}, \alpha, d) \dot{d}=0\end{array} \quad, \quad \forall \boldsymbol{x} \in \Omega\right.$ & $(20)$ \\
\hline yield surface & $f_{\mathrm{d}}(\varepsilon, \boldsymbol{p}, \alpha, d):=$ & $(21)$ \\
\hline \multicolumn{3}{|c|}{$-\mathrm{g}^{\prime}(d) \psi_{\mathrm{e}}(\boldsymbol{\varepsilon}-\boldsymbol{p})-\mathrm{h}^{\prime}(d) \psi_{\mathrm{p}}(\alpha, \nabla \alpha)$} \\
\hline & $-\frac{G_{\mathrm{c}}}{c_{\omega}}\left(\frac{\omega^{\prime}(d)}{\ell}-\ell \Delta d\right)-\mathrm{p}^{\prime}(d) \Delta_{\mathrm{p}}(\alpha)$ & \\
\hline $\begin{array}{l}\text { boundary } \\
\text { condition }\end{array}$ & $\nabla d \cdot \boldsymbol{n}=0, \quad \forall \boldsymbol{x} \in \partial \Omega$ & $(22)$ \\
\hline
\end{tabular}

Table 2: Governing equations and evolution laws deduced from the energetic formulation. 


\section{Overview of existing models}

Tab. 3 gives an overview of the existing models, presented with a unified notation and with the format induced by (12). Each model is presented in terms of the energy contributions $\mathbf{E}, \mathbf{H}, \mathbf{F}$ and $\mathbf{P}$. The next sections are devoted to the discussion of the different constitutive choices the investigated models rely on. However, some preliminary observations are immediately given:

1. Concerning $\mathbf{E}$ in (12), the models differ only for the choice of the degradation function $\mathrm{g}$, for which the key properties (3) are always fulfilled. Most models adopt the standard quadratic expression (4). Parametric cubic and non-linear degradation functions are considered by Alessi et al. [1, 2] and Borden et al. [9], respectively. The latter contains, as a limit case, the standard quadratic expression. Finally, a specific quadratic-like g, which also depends on the accumulated plastic strain, is considered by Ambati et al. [5]. The motivation behind each particular choice is outlined in Sections 3.1 and 3.4 .

2. Stronger differences concern the plastic isotropic hardening entry $\mathbf{H}$. The most general representation for $\psi_{\mathrm{p}}$ is considered by Miehe at al. [17], where the leading linear hardening term $\frac{1}{2} H \alpha^{2}$ is enriched by adding a nonlinear one and a non-local gradient contribution with its own length-scale parameter, as in (10). All other models consider at most linear hardening effects. Only Alessi et al. [1, 2, 3] do not consider plastic hardening effects, see Sec. 3.3. However a straightforward extension of their model by Ulloa et al. [20] does. In all models with the exceptions of Duda et al. [12] and Ambati et al. [5], plastic hardening is coupled with damage and the degradation multiplier $\mathrm{h}$ is typically taken identical to the function $\mathrm{g}$. Such a coupling has a strong impact on the evolution and interplay of damage and plasticity during the softening stage, as discussed in Sections 3.1, 3.2 and 4 .

3. In contrast to $\mathbf{H}$, the fracture energy entry $\mathbf{F}$ is quite standard for all the examined models. In all but one cases, $\Delta_{\mathrm{f}}$ is given by (7) combined with either AT-1 or AT-2, (8). The only exception, discussed in Sec. 3.5, is proposed in Miehe at al. [17], where the fracture energy term can formally be viewed as a two-parametric extension of the AT-2 model.

4. Finally, the fourth term $\mathbf{P}$ differs between the models only for the presence or not of the degradation function $\mathrm{p}(d)$ which indicates whether the plastic dissipation (11) is affected or not by the damage evolution. In the case of coupling, the corresponding degradation function $p$ is typically chosen identical to $g$. The only exception is in Alessi et al. [1,2,3], where a simple parametric expression allows to achieve different coupling orders, including the quadratic and uncoupled case, and to describe very different material behaviours. No coupling is assumed in Duda et al. [12] and Ambati et al. [5]. 


\begin{tabular}{|c|c|c|c|c|}
\hline model name & $\begin{array}{c}\mathbf{E} \\
\mathrm{g}(d) \psi_{\mathrm{e}}(\varepsilon-\boldsymbol{p})\end{array}$ & $\begin{array}{c}\mathbf{H} \\
\mathrm{h}(d) \psi_{\mathrm{p}}(\alpha, \nabla \alpha)\end{array}$ & $\begin{array}{c}\mathbf{F} \\
\Delta_{\mathrm{f}}(d, \nabla d)\end{array}$ & $\begin{array}{c}\mathbf{P} \\
\mathrm{p}(d) \Delta_{\mathrm{p}}(\alpha)\end{array}$ \\
\hline $\begin{array}{l}\text { Alessi et al. } \\
{[1,2,3]}\end{array}$ & $\begin{array}{c}\frac{(1-d)^{2}}{k-(k-1)(1-d)^{2}} \psi_{\mathrm{e}}(\boldsymbol{\varepsilon}-\boldsymbol{p}) \\
\text { with } k>0 . \text { For } k=1 \text { one recovers (4) }\end{array}$ & - & $\begin{array}{r}\frac{3 G_{\mathrm{c}}}{8}\left(\frac{d}{\ell}+\ell|\nabla d|^{2}\right) \\
\text { AT- } 1,(7)-(8)\end{array}$ & $\begin{array}{l}\quad(1-d)^{2 n} \sigma_{\mathrm{p}} \alpha \\
\text { with } n>0\end{array}$ \\
\hline $\begin{array}{l}\text { Borden et al. } \\
{[9]}\end{array}$ & $\begin{array}{c}\mathrm{g}(d) \psi_{\mathrm{e}}(\boldsymbol{\varepsilon}-\boldsymbol{p}) \\
\text { with } \mathrm{g}(d)=(1-d)^{2}(1+d(2-k)) \text { and } \\
k>0 . \text { For } k=2 \text { one recovers }(4)\end{array}$ & $\begin{array}{c}\mathrm{h}(d)\left(\frac{1}{2} H \alpha^{2}\right) \\
\text { with } \mathrm{h}(d) \equiv \mathrm{g}(d), \mathrm{g} \text { defined in } \mathbf{E}\end{array}$ & $\begin{array}{r}\frac{G_{\mathrm{c}}}{2}\left(\frac{d^{2}}{\ell}+\ell|\nabla d|^{2}\right) \\
\text { AT-2, (7)-(8) }\end{array}$ & $\begin{array}{l}\qquad \mathrm{p}(d) \sigma_{\mathrm{p}} \alpha \\
\text { with } \mathrm{p}(d) \equiv \mathrm{g}(d), \mathrm{g} \\
\text { defined in } \mathbf{E}\end{array}$ \\
\hline $\begin{array}{l}\text { Duda et al. } \\
{[12]}\end{array}$ & $(1-d)^{2} \psi_{\mathrm{e}}(\boldsymbol{\varepsilon}-\boldsymbol{p})$ & $\frac{1}{2} H \alpha^{2}$ & & $\sigma_{\mathrm{p}} \alpha$ \\
\hline $\begin{array}{l}\text { Kuhn et al. } \\
{[14]}\end{array}$ & & $(1-d)^{2}\left(\frac{1}{2} H \alpha^{2}\right)$ & & $(1-d)^{2} \sigma_{\mathrm{p}} \alpha$ \\
\hline $\begin{array}{l}\text { Miehe et al. } \\
{[17]}\end{array}$ & & $\begin{array}{l}(1-d)^{2}\left(\frac{1}{2} H \alpha^{2}+\mathrm{f}(\alpha)+\frac{1}{2} \ell_{\mathrm{p}}^{2}|\nabla \alpha|^{2}\right) \\
\text { with } \mathrm{f}(\alpha)=\left(\sigma_{\infty}-\sigma_{\mathrm{p}}\right)\left(\alpha+\frac{1}{\eta} e^{-\eta \alpha}\right) \\
\text { and } \eta>0, \sigma_{\infty}>\sigma_{\mathrm{p}}\end{array}$ & $\begin{array}{l}\left(1-(1-d)^{2}\right) w_{c}+\frac{w_{c}}{\xi} \ell\left(\frac{d^{2}}{\ell}+\ell|\nabla d|^{2}\right) \\
\text { with } w_{c}>0, \xi>0\end{array}$ & \\
\hline $\begin{array}{l}\text { Ambati et al. } \\
{[5]}\end{array}$ & $\begin{array}{l}\qquad(1-d)^{2 \mathrm{~b}(\alpha)} \psi_{\mathrm{e}}(\boldsymbol{\varepsilon}-\boldsymbol{p}) \\
\text { where } \mathrm{b}(\alpha)=\left(\alpha / \alpha_{\text {crit }}\right)^{m} \text {, with } \alpha_{\text {crit }}>0 \\
\text { and } m>0\end{array}$ & $\frac{1}{2} H \alpha^{2}$ & $\begin{array}{r}\frac{G_{\mathrm{c}}}{2}\left(\frac{d^{2}}{\ell}+\ell|\nabla d|^{2}\right) \\
\mathrm{AT}-2,(7)-(8)\end{array}$ & $\sigma_{\mathrm{p}} \alpha$ \\
\hline
\end{tabular}

Table 3: Overview of the total internal energy density $W_{\mathrm{PD}}$ for the compared models. The labels refer to (12). 


\subsection{On the lack of a damage-plastic coupling}

In Duda et al. [12], no coupling between damage and plasticity is prescribed, in the sense that the plastic yield condition (16) does not depend upon the damage variable whereas the damage yield condition (20) does not depend upon the plastic variable. Thus, the damage evolution is driven only by elastic strains and is governed by the classical brittle phase-field law. Therefore, the formulation in [12] is a model for "brittle fracture in elastic-plastic solids".

The coupling source for the model in Ambati et al. [5] relies only on the special degradation function of the elastic potential energy E whereas for the remaining models coupling effects originate from $\mathrm{H}$ and/or $\mathrm{P}$.

\subsection{On the identical degradation functions}

In the models of Borden et al. [9], Kuhn et al. [14] and Miehe at al. [17], all three degradation functions in $\mathbf{E}, \mathbf{H}$ and $\mathbf{P}$ are assumed identical, i.e. $\mathrm{g} \equiv \mathrm{h} \equiv \mathrm{p}$. After factoring them out in the plastic yield function $f_{\mathrm{p}}$, (18), one arrives at the representation

$$
f_{\mathrm{p}}(\boldsymbol{\sigma}, \alpha, d)=\mathrm{g}(d)\left(\left|\boldsymbol{\varepsilon}^{\prime}-\boldsymbol{p}\right|-\left(H \alpha+\sigma_{\mathrm{p}}\right)\right)
$$

with $\varepsilon^{\prime}$ as the deviatoric part of $\varepsilon$ and where non-linear hardening and gradient plastic terms are omitted. Thus, the evolution of the accumulated plastic strain becomes independent on the phase-field. This allows the straightforward use of standard elasto-plastic algorithms. As a side effect, $\alpha$ will grow approximatively with half the rate of $|\dot{\varepsilon}|$ even in regions where the material is already fully degraded, possibly causing convergence issues since $\varepsilon$ strongly localises and so must $\boldsymbol{p}$ and $\alpha$. Such behaviour is illustrated in Sec. 4. Note that $\mathrm{g} \equiv \mathrm{h} \equiv \mathrm{p}$ does not affect the damage yield function $f_{\mathrm{d}}$ in the way it happens for $f_{\mathrm{p}}$, meaning that the evolution of $d$ will still be affected by $\alpha$.

\subsection{On the lack of plastic hardening}

In the original model of Alessi et al. [1, 2, 3], no plastic hardening effects are considered. This allows for plastic strains to localise as Dirac's measures leading to the formation of shear bands. In such a case, the governing equations of Table 2 are complemented with the equations for the singular parts of displacement and the plastic strain fields, leading to a rigorous description of shear bands and therefore displacement jumps. This has been proved to be a key feature in describing different fracture cohesive responses. 


\subsection{Non-standard degradation functions}

Referring to (4) as the "standard" quadratic degradation function, nonstandard choices for $\mathrm{g}$ (yet fulfilling (3)), have been considered in the models of Alessi et al. [1, 2], Borden et al. [9] and Ambati et al. [5].

Alessi et al. $[1,2]$ considered the one-parametric non-linear degradation function

$$
\mathrm{g}(d):=\frac{(1-d)^{2}}{k-(k-1)(1-d)^{2}}, \quad k>0,
$$

which recovers (4) for $k=1$. The parameter $k$ controls the material response in the post-critical stage. In particular, as shown e.g. in [2] for the stressstrain uniaxial homogeneous response, a larger $k$ leads to a less steep softening curve, i.e. to a slower damage evolution.

The one-parametric cubic degradation function

$$
\mathrm{g}(d):=\mathrm{g}(d)=(1-d)^{2}(1+d(2-k)), \quad k>0,
$$

in [9] is adopted from Borden et al. [8], where its implications are discussed in detail. Unlike for the combination "quadratic g + AT-2", where damage starts at zero loading, the combination "quadratic g + AT-1" has a linearly elastic stage up to the peak stress. However, the boundness of $d \in[0,1]$, which automatically holds for the "quadratic g + AT-2" combination, is no longer fulfilled. This leads to the need for constrained minimization algorithms. The cubic function proposed in [8], with an extremely small $k$, used with AT-2, retains the presence of the elastic limit and the boundedness of $d$.

Finally, a specific non-linear function

$$
\mathrm{g}(d, \alpha):=(1-d)^{2\left(\alpha / \alpha_{\text {crit. }}\right)^{m}}, \quad m>0,
$$

is proposed in [5]. Its introduction serves the purpose of realizing the plasticdamage coupling, as already explained in Sec. 3.1. With the above g, $\alpha$ explicitly appears in the damage evolution equation and fracture is triggered by the accumulation of the ductile damage once the threshold $\alpha_{\text {crit. }}$ is reached. The parameter $m$ provides additional flexibility: the increase of $m$ slows down the accumulation of damage before reaching $\alpha_{\text {crit. }}$ and accelerates it when $\alpha_{\text {crit. }}$. is exceeded, see [5] for a complete numerical assessment.

\subsection{Non-standard fracture dissipation term}

A feature of the model in [17] is the damage dissipation density function

$$
\Delta_{\mathrm{f}}:=(1-\mathrm{g}(d)) w_{c}+\frac{w_{c}}{\xi} \ell\left(\frac{d^{2}}{\ell}+\ell|\nabla d|^{2}\right), \quad w_{c}>0, \xi>0,
$$


The parameter $w_{c}$ governs the onset of fracture, whereas $\xi$ controls the slope of softening. The proposed $\Delta_{\mathrm{f}}$ can formally be viewed as an extension of the AT-2 model. An interesting question is that of the relation between $w_{c}$ and $G_{\mathrm{c}}$. Being $\xi$ dimensionless, $w_{c}$ has the dimension of $G_{\mathrm{c}} / \ell$. Our idea of deriving the explicit dependency of $w_{c}$ on $G_{\mathrm{c}}$ is to rescale $\ell=\sqrt{2} \tilde{\ell}$ and collapse the two terms in $\Delta_{\mathrm{f}}$. This yields the representation

$$
\Delta_{\mathrm{f}}=2 \frac{w_{c}}{\xi} \tilde{\ell}\left(\frac{2 \xi d-(\xi-1) d^{2}}{2 \tilde{\ell}}+\tilde{\ell}|\nabla d|^{2}\right) .
$$

Setting $\xi=1$, the above $\Delta_{\mathrm{f}}$ resembles AT-1 provided $w_{c}=3 / 16 G_{\mathrm{c}} / \tilde{\ell}$.

\subsection{On the damage irreversibility}

The damage irreversibility is imposed by requiring $\dot{d} \geq 0$. Already for brittle fracture, this condition is known to lead to a box-constrained optimization problem for the functional $\mathcal{W}$. To avoid this for the brittle case, the history variable

$$
\mathcal{H}_{t}:=\max _{s \leq t} \psi_{\mathrm{e}}\left(\varepsilon_{s}\right)
$$

which records the maximal undegraded elastic density energy obtained in a loading process, can be introduced in the evolution equation for $d$, as originally proposed in [16]. This weak enforcement of irreversibility can only be used with models where $d$ starts to evolve as soon as the material is loaded, as the AT-2 model but not AT-1. Another approach consists on imposing the irreversibility only for totally broken material points, i.e. where $d=1$, as proposed e.g. in [10].

In ductile phase-field models, the above definition of $\mathcal{H}_{t}$ appears in the formulations of Duda et al. [12], Ambati et al. [5] and Borden et al. [9]. A more sophisticated expression for $\mathcal{H}_{t}$ is designed in Miehe et al. [17], see also Sec. 3.7. The formulation of Kuhn et al. [14] uses the irreversibility idea from [10]. The treatment of $\dot{d} \geq 0$ through optimization algorithms is adopted in Alessi et al. $[1,2,3]$.

\subsection{Parameters and thresholds}

The capability to tailor the constitutive response to match experimental data is an important asset of any formulation. The role of some constitutive parameters and thresholds, designed for this purpose, has been already discussed in Sections 3.4 and 3.5, limited to the formulations of Alessi et al. [1, 2, 3], Borden et al. [9], Miehe et al. [17] and Ambati et al. [5]. As follows, we focus on additional quantities introduced in some formulations at a later stage of 
the model derivation. This holds for the models of Borden et al. [9] and Miehe et al. [17]. In [9], the evolution equation for $d$ (with the identity $\mathrm{h} \equiv \mathrm{p}$ ) reads

$$
\mathrm{g}^{\prime}(d) \psi_{\mathrm{e}}+\mathrm{h}^{\prime}(d)\left(\psi_{\mathrm{p}}+\Delta_{\mathrm{p}}\right)+\frac{G_{\mathrm{c}}}{\ell}\left(d-\ell^{2} \Delta d\right)=0 .
$$

This expression is furthermore modified by introducing (i) the elasticity-based history variable $\mathcal{H}_{t}$ for irreversibility, (ii) a plastic work threshold $W_{0}$, and (iii) two parameters $\beta_{e}, \beta_{p} \in[0,1]$ to weigh the influence of damage on the elastic strain energy and plastic work. The resulting equation becomes

$$
\beta_{e} \mathrm{~g}^{\prime}(d) \mathcal{H}_{t}+\beta_{p} \mathrm{~h}^{\prime}(d)\left\langle\psi_{\mathrm{p}}+\Delta_{\mathrm{p}}-W_{0}\right\rangle_{+}+\frac{G_{\mathrm{c}}}{\ell}\left(d-\ell^{2} \Delta d\right)=0,
$$

where $\langle a\rangle_{+}:=\max (0, a)$. In [17] the evolution equation for $d$ is equipped with the history variable $\mathcal{H}_{c}:=\max _{s \in[0, t]}\left\langle\psi_{\mathrm{e}}+\psi_{\mathrm{p}}+\Delta_{\mathrm{p}}-w_{c}\right\rangle_{+}$, yielding

$$
-2(1-d) \mathcal{H}_{c}+2 \frac{w_{c}}{\xi}\left(d-\ell^{2} \Delta d\right)=0 .
$$

\subsection{Variational (in) consistency}

Almost all models in Tab. 3 fit in the variational framework. However, as discussed in the previous sections, the introduction of various indicator and ramp functions, tuning parameters and threshold values into the strong form equations to enable a greater flexibility in the model response inevitably leads to the loss of variational consistency. This holds for the works of Duda et al. [12], Ambati et al. [5], Borden et al. [9] and Miehe at al. [17]. These models are therefore semi-variational. Only the formulations of Alessi et al. [1, 2, 3] and Kuhn et al. [14] retain a truly variational nature. However, only the former model is rich enough to capture a variety of anticipated ductile and cohesive fracture responses.

\subsection{Additive split of the elastic energy density function}

Our last comment concerns the so-called tension-compression additive split of the elastic energy density function $\psi_{\mathrm{e}}$, first discussed by $[7,16]$, which is incorporated in several models but omitted for the sake of brevity in Tab. 3 . In general, the split representation $\mathrm{g}(d) \psi_{\mathrm{e}}^{+}+\psi_{\mathrm{e}}^{-}$for $\mathbf{E}$, with $\psi_{\mathrm{e}}^{+}$and $\psi_{\mathrm{e}}^{-}$as a "sort of" tensile and compressive parts of $\psi_{\mathrm{e}}$, respectively, is present in all formulations except for the one by Alessi et al. [1, 2, 3] and Kuhn et al. [14]. 


\section{Numerical comparisons}

This section aims at highlighting the salient features of the models by presenting a short numerical comparative survey of a uniaxial tension test, consisting of a bar of length $L$, clamped on its left end, with a monotonically increasing displacement $\bar{u}$ prescribed on its right end. The adopted material parameters, taken from [9], are the following: $\mathrm{E}_{0}=68.8 \mathrm{GPa}, \sigma_{\mathrm{p}}=320 \mathrm{MPa}$, $H=688 \mathrm{MPa}$, and $G_{\mathrm{c}}=138 \mathrm{MPam}$. Such parameters correspond to a ductile fracture response with an elastic stage, a linear-hardening plastic stage and a softening/fracturing stage.

Both homogeneous and non-homogeneous responses are investigated. The former, captured with a short bar length, highlights the underlying material response, whereas the latter evidences the localisation/fracture process. For the non-homogeneous response, the value of the bar length $L=L_{\mathrm{n}}$ is chosen sufficiently large to allow the development of a full localisation but not too large in order to avoid snap-back effects. The influence of the bar length on the non-homogeneous global response is discussed, for instance, in [3]. Homogeneous responses are obtained numerically as well by taking $L=0.1 L_{\mathrm{n}}$.

Since the damage threshold stress for the phase-field model (7) directly depends on the internal length, even for an homogeneous evolution of the damage variable such as, for instance, during the hardening stage, different internal lengths are chosen for the models in order to obtain (except for Duda et al.) approximately the same peak stress and its corresponding limit strain. Setting $\ell=L_{\mathrm{n}} / 30$, different bar lengths $L_{\mathrm{n}}$ are considered.

The finite element mesh is uniform with element size $h=L / 500$. A staggered numerical solution scheme [5] is adopted. For the present simple test, irreversibility is not taken into account.

As follows, the numerical results for each model are presented. The homogeneous $(\mathrm{H})$ and non-homogeneous $(\mathrm{NH})$ responses of each model are visualised by four plots: (a) stress-mean strain $\mathrm{H}$ and $\mathrm{NH}$ responses, (b) accumulated plastic strain and damage profiles for the NH response, (c) plastic strain and damage evolutions for the $\mathrm{H}$ response, (d) maximum plastic strain and maximum damage evolutions for the $\mathrm{NH}$ response.

In the results presented in Fig. 2 and Fig. 3, the model by Alessi et al. is enriched by a linear hardening term, not present in the original formulation, according to [20]. Hence, plastic strains cannot localise as a Dirac measure anymore, Sec. 3.3. Nevertheless they localise in a narrower band than the damage. The main difference between Fig. 2 and Fig. 3 is the different value of the exponent of the degradation function $\mathrm{p}(d)$, Tab. 3. During the softening stage, for $n=1$ the stress approaches asymptotically zero (Barenblatt's cohesive behaviour), whereas for $n=0.5$ it vanishes at a finite strain value (Dugdale's cohesive behaviour). This different behaviour is due to the fact that in the former case plasticity continues to evolve during the softening stage, whereas in the latter it does not. 
Plastic strains evolve in a localised manner during the softening stage also in Miehe et al. (Fig. 8) (whose response is very similar to Alessi et al. in Fig. 2), in Borden et al. (Fig. 4) and in Kuhn et al. (Fig. 6). Conversely, plastic strains stop their evolution immediately after the peak stress in Duda et al. (Fig. 7) and Ambati et al. (Fig. 10), since here the plastic evolution is associated to a stress-hardening response.

In the global response, an asymptotically vanishing stress in the fracturing stage is observed in Alessi et al. (Fig. 3), Borden et al. (Fig. 4), Kuhn et al. (Fig. 6) and Ambati et al. (Fig. 10). Within the last model no plastic strain localisation is observed while for the remaining models a true fracture with vanishing stress is achieved for a finite strain value.

The plastic hardening-damaging softening transition is smooth for Borden et al. (Fig. 4) and Kuhn et al. (Fig. 6) and sharp in all other cases. In Kuhn et al. Fig. 6, no stress-hardening effects are observed, which is in contrast with the experimental evidence.

Some models (Duda et al., Kuhn et al. and Ambati et al.) show after the peak stress an homogeneous damage evolution and only successively a damage localisation evolution associated to a steeper global response curve. Such material bifurcation, associated to the choice of an "intermediate" bar length, is very well investigated and explained in [19].

Damage increases very fast during the plastic-hardening stage in Kuhn et al. and Ambati et al. while it increases imperceptibly in all other models, except for Alessi et al. where it does not evolve at all, even during the elastic stage, Sec. 2.2. In addition, Duda et al. model has a significant damage evolution even during the elastic stage.

Fig. 5 highlights the role of $W_{0}$ in (25), see Sec. 3.7, which essentially tunes the value of the peak stress. A similar control is performed by the parameter $\alpha_{\text {crit }}$ in Ambati et al., see Sec. 3.4. In addition, Fig. 9 highlights the role of $\xi$ in (24), see Sec. 3.5, which tunes the concavity of the global response in the softening stage. 


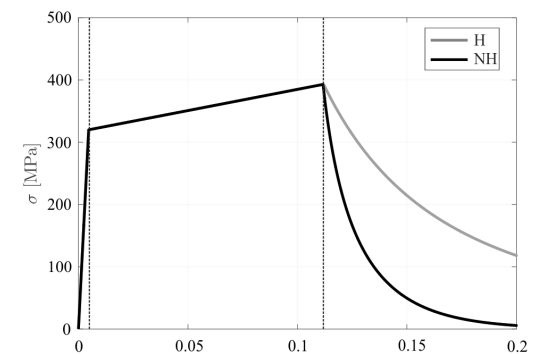

(a)

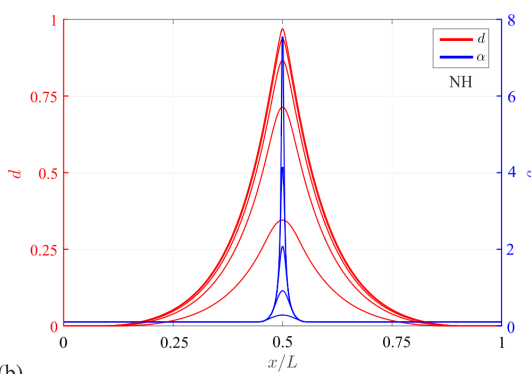

(b)

Fig. 2: Alessi et al. Homogeneous and non-homogeneous responses for a bar length $L_{n}=10 \mathrm{~mm}$ and $n=1$.

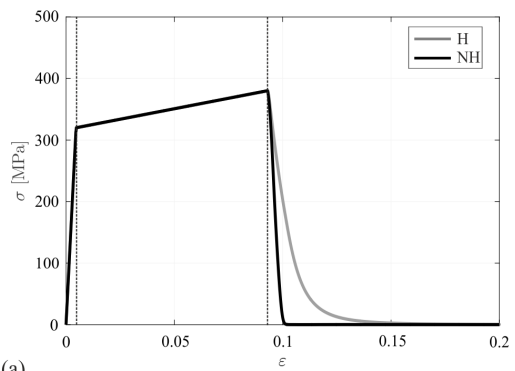

(a)

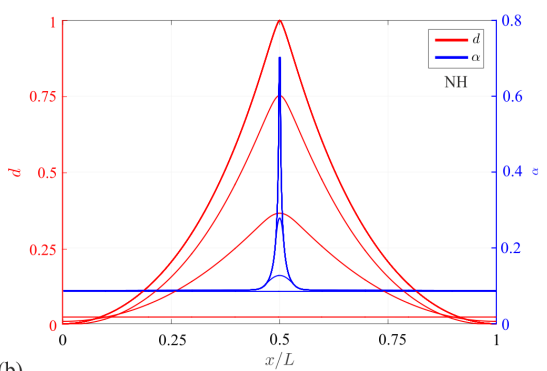

(b)

Fig. 3: Alessi et al. Homogeneous and non-homogeneous responses for a bar length $L_{n}=22 \mathrm{~mm}$ and $n=0.5$.

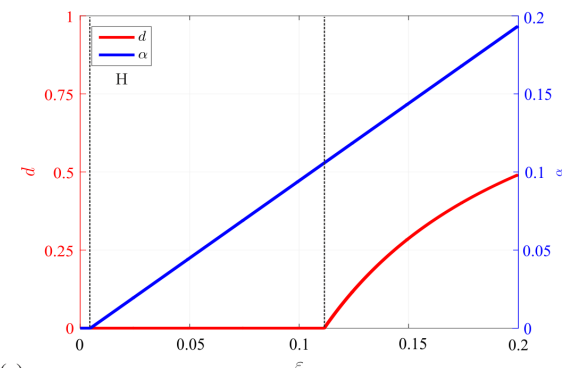

(c)

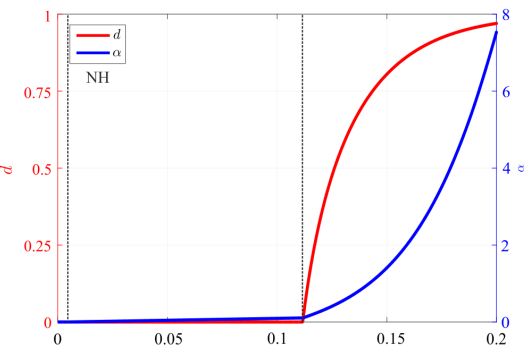

(d)

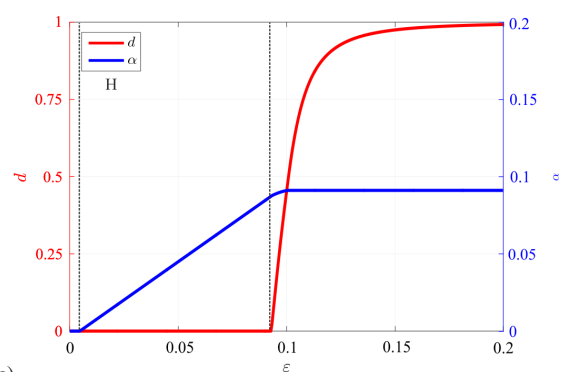

(c)

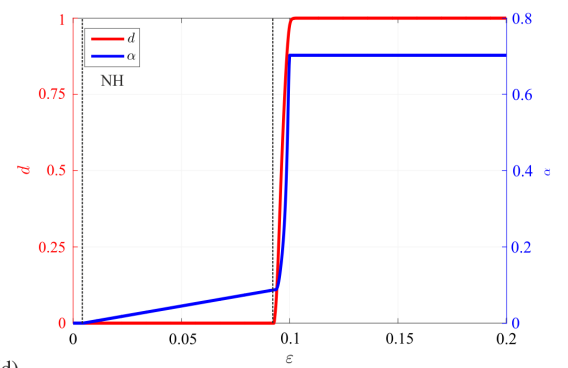

(d) 


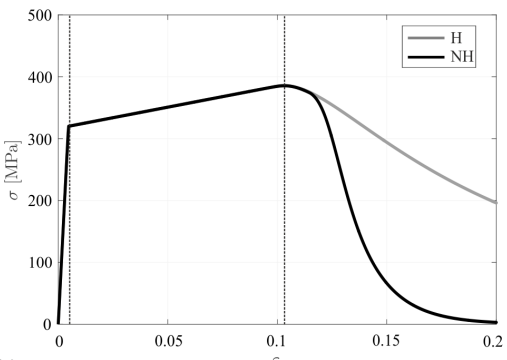

(a)

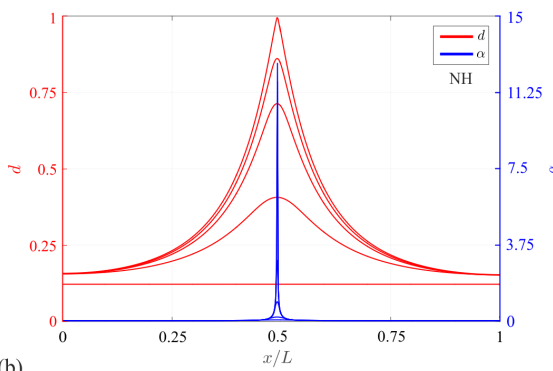

(b)

Fig. 4: Borden et al. Homogeneous and non-homogeneous responses for a bar length $L_{n}=10 \mathrm{~mm}$ and $W_{0}=0$.

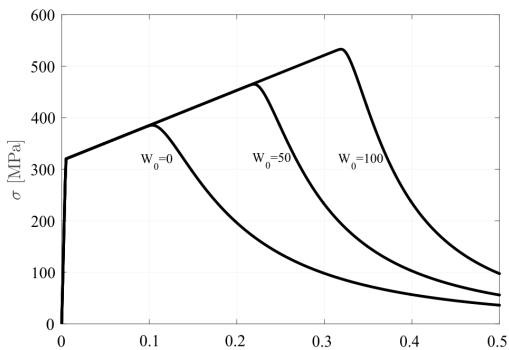

(a)

Fig. 5: Borden et al. Effect of $W_{0}$ on the homogeneous response
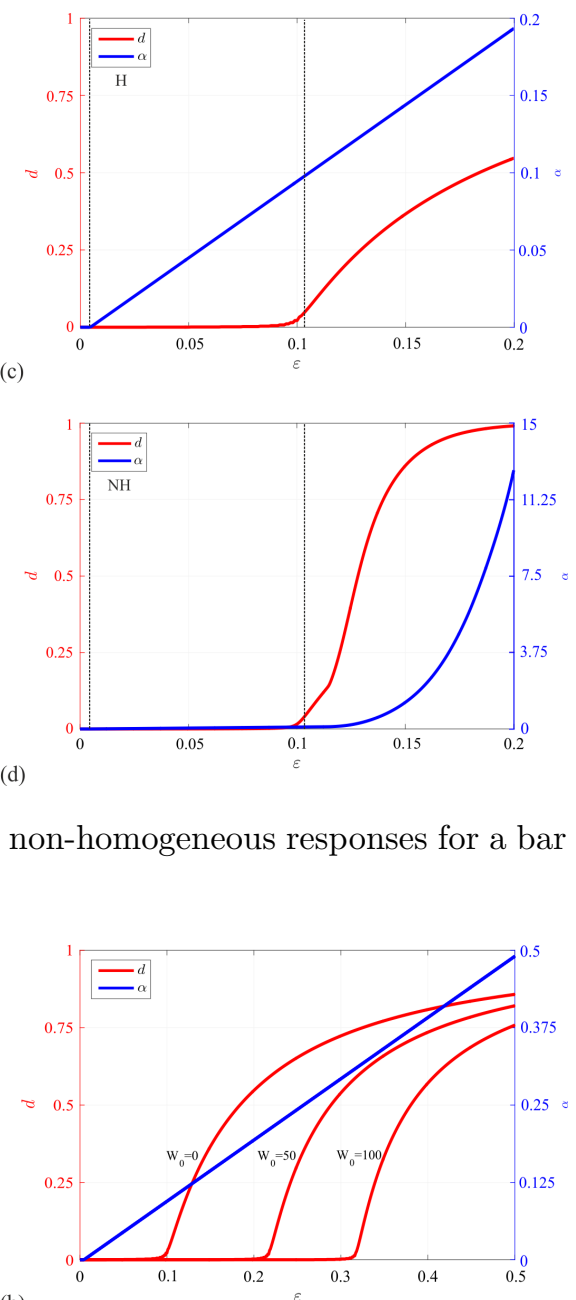

(b) 


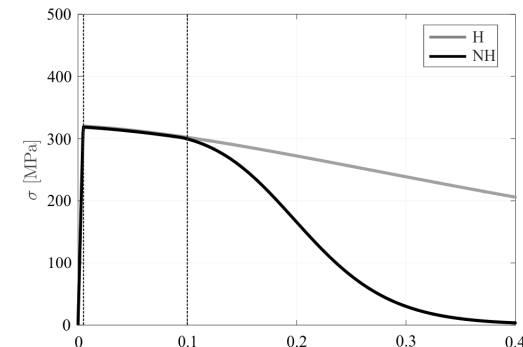

(a)

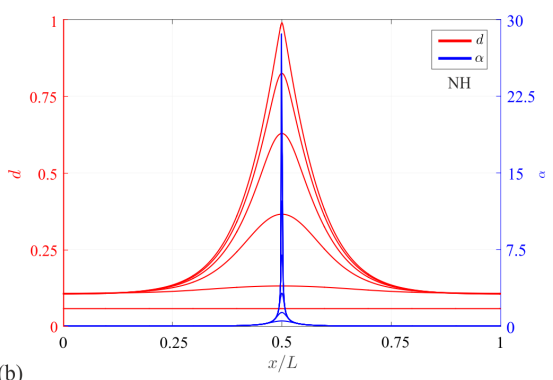

(b)

Fig. 6: Kuhn et al. Homogeneous and non-homogeneous responses for a bar length $L_{n}=3.5 \mathrm{~mm}$.

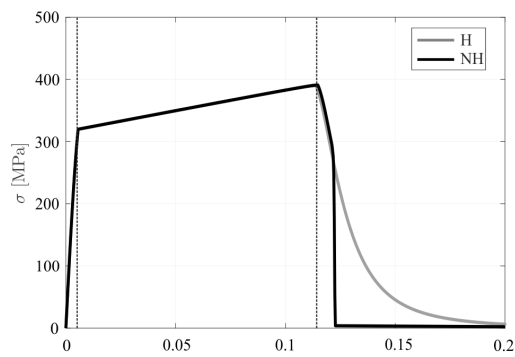

(a)

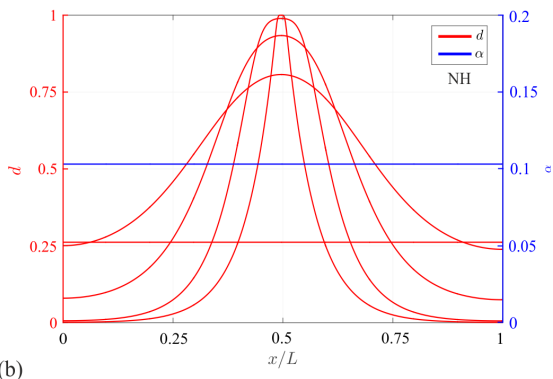

(c)
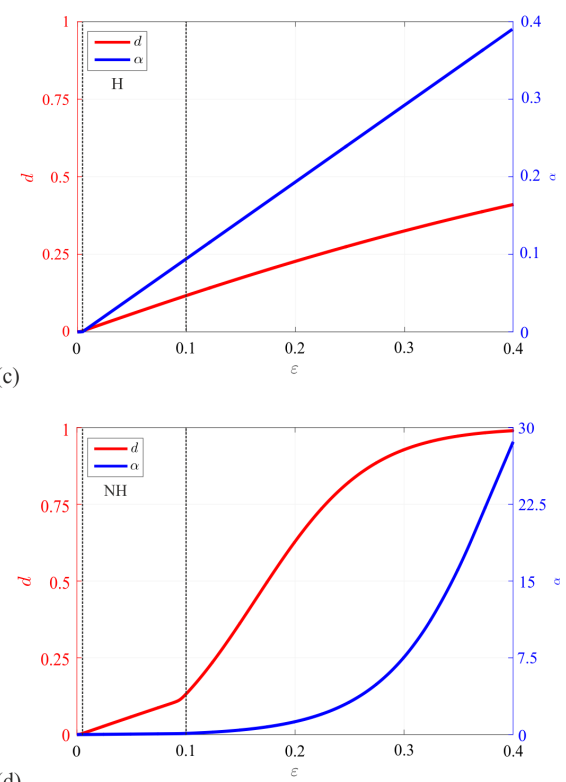

(d) 


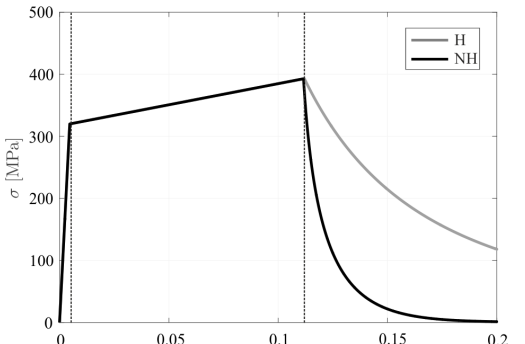

(a)

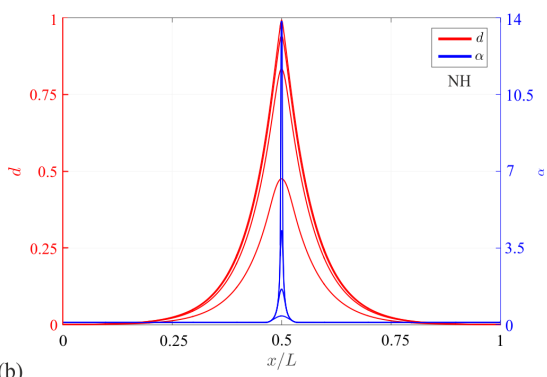

(b)

Fig. 8: Miehe et al. homogeneous and non-homogeneous responses for a bar length $L_{n}=10 \mathrm{~mm}, w_{c}=\frac{3 G_{c}}{16 \ell}$ and $\xi=1$.

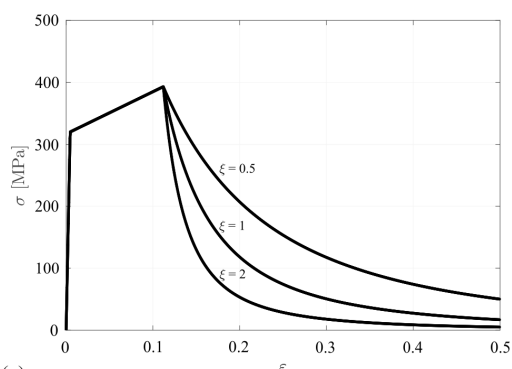

(a)

Fig. 9: Miehe et al. Effect of $\xi$ on the homogeneous response.

(c)
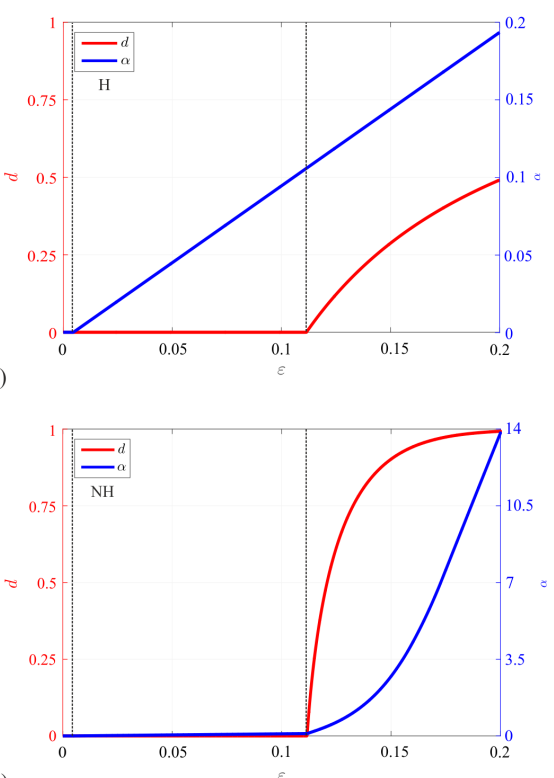

(d)

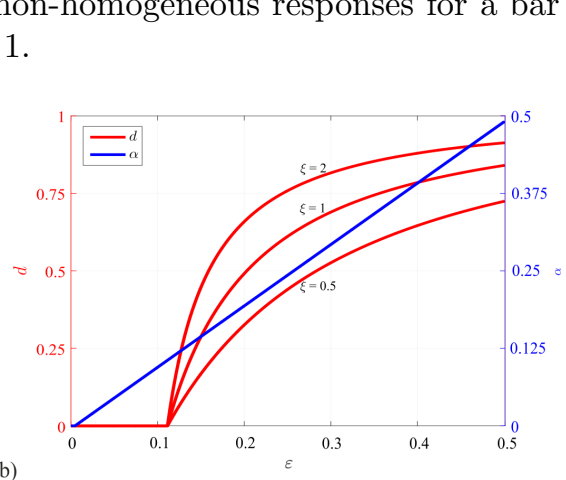

(b) 


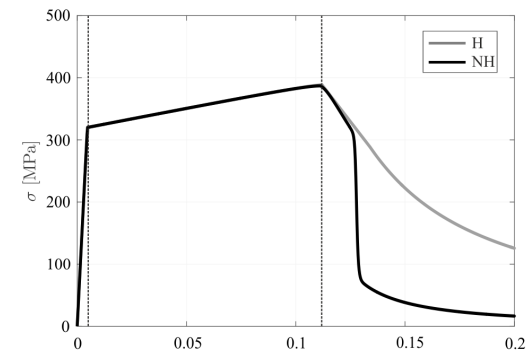

(a)

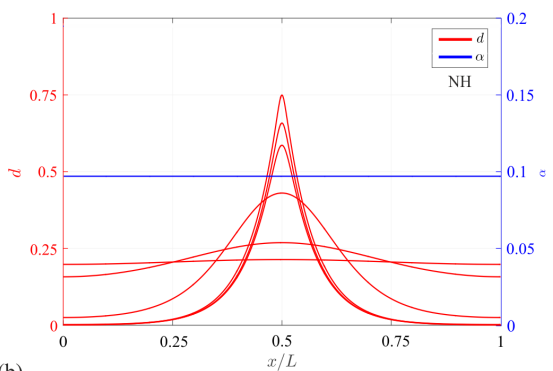

(b)

Fig. 10: Ambati et al. homogeneous and non-homogeneous responses for a bar length $L_{n}=10 \mathrm{~mm}$, and $\alpha_{\text {crit }}=0.025$.

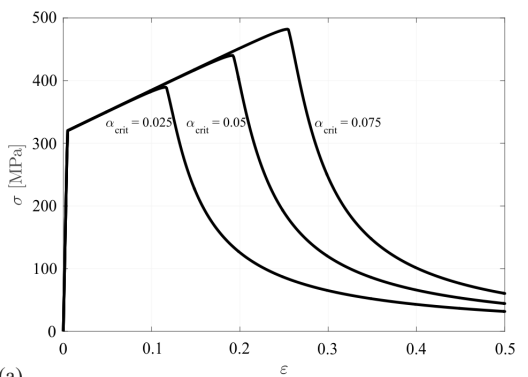

(a)

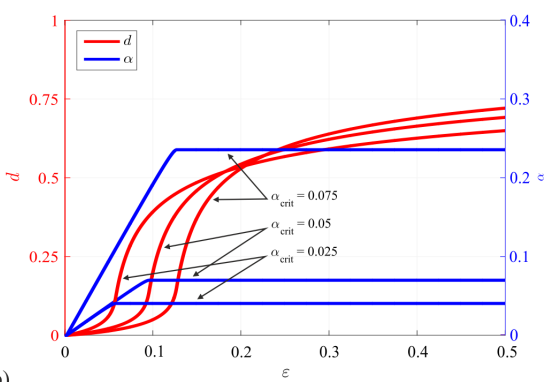

Fig. 11: Ambati et al. Effect of $\alpha_{\text {crit }}$ on the homogeneous response. 


\section{Concluding remarks}

We reported a critical comparative review of existing phase-field models for fracture in elasto-plastic materials. All models can be cast in the same variational framework and differ mainly by the choices of the damage dissipation term, by the degradation function(s) as well as by the way the coupling between plasticity and damage is realized. The models in "basic" form (i.e. with the minimum possible amount of tailorable parameters, if any) retain a variational nature but are not always able to reproduce an experimental response due to lack of flexibility. More elaborated versions with additional ingredients such as thresholds and indicator functions gain flexibility but may loose the variational nature. Two main categories of models can be identified based on whether the plastic hardening variable stops or continues its evolution once damage is triggered. The behavior of real materials in this respect would be easily identified by analyzing the unloading response in uniaxial stress-strain curves. Finally, more sophisticated aspects of the phenomenology such as those examined in [5] as well as triaxiality effects remained out of the scope of this paper and should be accounted for in future work.

Acknowledgements The authors would like to acknowledge the support of the DAAD through the project "Variational approach to fatigue phenomena with phase-field models".

\section{References}

[1] Alessi R, Marigo JJ, Vidoli S (2014) Gradient Damage Models Coupled with Plasticity and Nucleation of Cohesive Cracks. Archive for Rational Mechanics and Analysis 214(2):575-615

[2] Alessi R, Marigo JJ, Vidoli S (2015) Gradient damage models coupled with plasticity: Variational formulation and main properties. Mechanics of Materials 80(Part B):351-367

[3] Alessi R, Marigo JJ, Maurini C, Vidoli S (2017) Coupling damage and plasticity for a phase-field regularisation of brittle, cohesive and ductile fracture: one-dimensional examples. International Journal of Mechanical Sciences Submitted

[4] Ambati M, Gerasimov T, De Lorenzis L (2015) A review on phase-field models of brittle fracture and a new fast hybrid formulation. Computational Mechanics 55:383-405

[5] Ambati M, Gerasimov T, De Lorenzis L (2015) Phase-field modeling of ductile fracture. Computational Mechanics 55(5):1017-1040

[6] Ambrosio L, Tortorelli VM (1990) Approximation of functional depending on jumps by elliptic functional via Gamma-convergence. Communications on Pure and Applied Mathematics 43(8):999-1036 
[7] Amor H, Marigo JJ, Maurini C (2009) Regularized formulation of the variational brittle fracture with unilateral contact: Numerical experiments. Journal of the Mechanics and Physics of Solids 57(8):1209-1229

[8] Borden MJ, Verhoosel CV, Scott MA, Hughes TJR, Landis CM (2012) A phase-field description of dynamic brittle fracture. Computer Methods in Applied Mechanics and Engineering 217:77-95

[9] Borden MJ, Hughes TJR, Landis CM, Anvari A, Lee IJ (2016) A phasefield formulation for fracture in ductile materials: Finite deformation balance law derivation, plastic degradation, and stress triaxiality effects. Computer Methods in Applied Mechanics and Engineering 312:130-166

[10] Bourdin B, Francfort GA, Marigo JJ (2008) The Variational Approach to Fracture. Journal of Elasticity 91(1):5-148

[11] Dal Maso G, Orlando G, Toader R (2016) Fracture models for elastoplastic materials as limits of gradient damage models coupled with plasticity: the antiplane case. Calc Var Partial Differential Equations 55(3):45

[12] Duda FP, Ciarbonetti A, Sánchez PJ, Huespe AE (2015) A phasefield/gradient damage model for brittle fracture in elastic-plastic solids. International Journal of Plasticity 65:269-296

[13] Francfort GA, Marigo JJ (1998) Revisiting brittle fracture as an energy minimization problem. Journal of the Mechanics and Physics of Solids 46(8):1319-1342

[14] Kuhn C, Noll T, Müller R (2016) On phase field modeling of ductile fracture. GAMM-Mitteilungen 39(1):35-54

[15] Marigo JJ, Maurini C, Pham K (2016) An overview of the modelling of fracture by gradient damage models. Meccanica 51(12):3107-3128

[16] Miehe C, Welschinger F, Hofacker M (2010) Thermodynamically consistent phase-field models of fracture: Variational principles and multi-field FE implementations. International Journal for Numerical Methods in Engineering 83(10):1273-1311

[17] Miehe C, Teichtmeister S, Aldakheel F (2016) Phase-field modelling of ductile fracture: a variational gradient-extended plasticity-damage theory and its micromorphic regularization. Philosophical transactions Series A, Mathematical, physical, and engineering sciences 374(2066)

[18] Mielke A, Roubíček T (2015) Rate-Independent Systems: Theory and Application. Springer

[19] Pham K, Marigo JJ, Maurini C (2011) The issues of the uniqueness and the stability of the homogeneous response in uniaxial tests with gradient damage models. Journal of the Mechanics and Physics of Solids 59(6):1163-1190

[20] Ulloa J, Rodríguez P, Samaniego E (2016) On the modeling of dissipative mechanisms in a ductile softening bar. Journal of Mechanics of Materials and Structures 11(4):463-490 